\title{
Irrelevant, incidental and core features in the retrograde amnesia associated with Korsakoff's psychosis: a review
}

\author{
P.R. Meudell \\ Department of Psychology, University of Manchester, Manchester M13 9PL, UK
}

\begin{abstract}
A brief review of the literature on retrograde amnesia in Korsakoff's syndrome is presented. Various explanations of the phenomenon are discussed including the notions that it results from the effects of "state- dependency", that it occurs as a result of a progressive learning problem and that it arises through a failure in contextual processing. None of these hypotheses can satisfactorily account for the length and temporal gradient of alcoholic amnesics retrograde amnesia. Although some evidence points towards the hypothesis that anterograde and retrograde amnesia might result from separate and independent impairments, this view is presently unproven and leaves open what causes the form and duration of Korsakoffs retrograde amnesia.
\end{abstract}

Keywords: Korsakoff - Memory - Retrograde amnesia

\section{INTRODUCTION}

The Wernicke-Korsakoff syndrome comprises a number of neurological deficits. From the neuropsychological perspective, however, it is the memory and related impairments which are of central clinical and theoretical concern.

Korsakoff amnesics can have normal intelligence as assessed by the WAIS and similar tests and appear to have normal linguistic functions. Whilst the major deficit is one of memory this impairment is far from total. Short term memory-as assessed by digit span and similar procedures - is quite normal and a range of perceptual and motor skills can be acquired and retained by amnesics as effectively as normal controls. Furthermore, these patients can show normal "priming" to previously acquired information: that is, whilst the Korsakoff amnesic might not be able to consciously recall or recognise information to which he has recently been exposed, some memory for the experience appears to be stored in so far as it can affect performance on non-memory related tasks. The Claparede phenomenon is an illustrative anecdotal example. (See Mayes, 1988 for a review of all these aspects of preserved in function in amnesia.)

In the face of these preserved intellectual and mnemonic abilities, Korsakoff amnesics show a profound problem in learning new information involving day-today personal experiences (anterograde amnesia) and in recollecting information acquired and stored in earlier life (retrograde amnesia) before their memory problems were clearly apparent.

This note reviews some of the issues surrounding recall of remote memories in Korsakoff amnesics and examines the potential linkages between explanations of anterograde amnesia and the form and duration of retrograde amnesia.

\section{METHODOLOGICAL AND RELATED ISSUES}

The failure on the part of patients to recall or recognise events from their past-putatively before the onset of their anterograde amnesia-is of considerable theoretical importance. In fact, retrograde amnesia is a phenomenon that continues to challenge all theories of normal human memory. There are a number of questions relating to the retrograde amnesia but this contribution addresses some of the issues in the area that directly addresses remote memory problems specifically observed in alcoholic Korsakoff patients.

One major factor is how remote memories are to be properly assessed: another is exactly which type of memories should be sampled. Ever since Ribot (1882) formulated the notion that older memories tend to be less vulnerable to disruption the problem of controlling for differential rehearsal or review of old compared to more recently acquired memories has been raised. Solutions to the problem have been varied. Many (e.g. Sanders and Warrington, 1971; Snowden, 1983) have relied upon questionnaire techniques about public events. By selecting events that were salient for only a specified and finite period of time, problems of potentially greater review of older memories have been sought to be overcome. However, two memories may be equally salient and so may be rehearsed or reviewed at the same rate but, necessarily, the 
older one will have been rehearsed more. In principle, therefore, either the amount or the rate of rehearsal can be controlled and either variable could determine what makes memories invulnerable to amnesia; in practice, it is probably easier to control for the rate of rehearsal.

Others have sought to manipulate the "difficulty" of items in "public event" questionnaires directly. Albert $e t$ al. (1979) found items that were "easy" (operationally defined as those items that more than $50 \%$ of an independent normal control group could recall or recognise) and "difficult" (those questionnaire items that at least 50\% of this group of people could not recall and/or recognise). Squire (e.g. Squire and Cohen, 1984) has produced a questionnaire about TV programs that were "aired" for a very restricted period. None of these methods is entirely satisfactory but they do share the advantage that accuracy of recall for the "public" events being assessed is at least verifiable (see Weiskrantz, 1985 for a review).

Verifiability of the accuracy of memory is not generally available in the case of memories of a more autobiographical type. Autobiographical memories relate to those personal (and more or less unique and idiosyncratic) memories that we all possess about our own experiences (Crovitz and Schiffman, 1974; Zola-Morgan et al., 1983; Cermak and O'Connor, 1983; Baddeley and Wilson, 1986; Warringon and McCarthy, 1988). Such knowledge has been tapped by asking normal and amnesic patients to produce personal memories in response to a target stimulus. Typically, people are presented with a word and asked to recall discrete episodes from their past that are associated with that item. Usually (but not necessarily always) the data so obtained cannot be checked for accuracy and the measure of memory derived typically relates to the sheer number of items produced in response to a cue and/or the quality or richness of the recall protocol.

It is a matter of some theoretical as well as practical importance whether memory for public events tends to be compromised to the same extent and in the same way as memory for autobiographical knowledge. Unfortunately, little is known about these relationships and correlation studies amongst the same groups of patients on both types of test could be informative. In this connection, however, it is interesting to note that MacKinnon and Squire (1989) have recently shown that amongst a group of five memory impaired patients, those who had the most difficulty recollecting autobiographical details were also those who performed poorly on tests of fact memory: this suggests that memory for more or less public events could be mediated by the same system that sustains more personal memories.

Another problem is, granting adequate mechanisms for "equating" for differential rehearsal and review of memories, how is "severity" of retrograde amnesia to be characterised? Severity of retrograde amnesia could be measured in terms of the duration affected or in terms of degree of disruption or indeed, of both these measures. Which could be the appropriate measure clearly depends upon potential theoretical views about what causes the retrograde amnesia.

\section{THE FORM OF RETROGRADE AMNESIA IN KORSAKOFFS PSYCHOSIS}

Questionnaire studies have tended to conform the clinical impressions that (a) the retrograde amnesia in Korsakoffs is of extremely long duration-extending to decades in many instances and (b) that there is a marked temporal gradient with older memories showing greater preservation than most recent memories: similar conclusions have been reached when autobiographical details have been requested of patients (Meudell et al., 1980; Albert et al, 1981; Kopelman, 1989; Gade and Mortensen, 1990).

The extremely long form of retrograde amnesia in Korsakoffs contrast sharply with more temporally limited types noted in amnesics of other (non alcoholic etiologies). Thus H.M. (Marslen-Wilson and Teubar, 1975), N.A. (Squire and Slater, 1978) and R.B. (Zola-Morgan et al., 1986) all have retrograde amnesias that extend to only a few years at most. Although recent data (e.g. Ogden and Corkin, 1991) have suggested that H.M.'s retrograde amnesia might extend back a decade or more (to the time when he became epileptic), it is still a much shorter duration than that shown in most Korsakoffs and, further, there is uncertainty as to what components should be attributable to epileptic attacks or to drug treatments. Similarly, patients receiving ECT for depression show a relatively mild deficit in recall of remote memories (Squire and Cohen, 1984). One explanation for this difference between Korsakoffs and patients of other etiologies is simply that the magnitude of the observed retrograde amnesia is a function of the overall severity of the amnesia of any individual patient (Weiskrantz, 1985). Put differently, and assuming a common factor in the causation of retrograde and anterograde amnesias, those patients with severe anterograde problems might also be expected to show severe retrograde problems and vice versa. Although this is clearly a possibility, in fact amongst Korsakoffs, severity of retrograde amnesia and severity anterograde amnesia tend not to correlate highly (Shimamura and Squire, 1986; Kopelman, 1989). On a related point, we (Mayes et al., 1988) observed that, for two of our Korsakoffs who came to post mortem, the one with the severest anterograde problems had the milder retrograde amnesia. Further, although N.A. probably has a mild anterograde amnesia, this could hardly be said of H.M. The apparent independence of anterograde and retrograde severity provides weak support for the idea that the two impairments might have different explanations and this issue will be taken up in a later section. 
An alternative explanation for the length of the retrograde amnesia in Korsakoffs relates to etiological factors. Specifically, it has been suggested by a number of workers that the prolonged period of alcohol abuse involved before a Wernicke-Korsakoff incident might not be without effects upon memory.

There have been three forms of this hypothesis.

The first is that a form of "state dependency" might be implicated with alcoholic Korsakoffs (Kinsbourne and Wood, 1982). That is, since it is known that recall of information is critically dependent upon the re-instatement of original learning conditions (e.g. Eich, 1980), perhaps the fact that Korsakoffs acquired much information whilst intoxicated means that, when tested sober, their recall is especially poor. Whilst an attractive notion (and one that has interesting therapeutic implications for methods of recovering apparently unavailable memories in alcoholic amnesics), it is probably wrong. This is so since the effects contextual and state dependent shifts tend to apply in any significant degree only to free or cued recall and not to recognition: yet Korsakoff amnesics show profound retrograde amnesia however remote memories are tested, including cued recall prompts as well as recognition tests. Further, state dependent effects are hardly of a sufficient magnitude to be able to realistically explain the severity of some Korsakoffs retrograde amnesia.

The second version of the hypothesis is that a pre-morbid preoccuption with alcohol might render the alcoholics less likely to become involved (through the media and so forth) with day to day newsworthy events than would ordinary people (Albert et al., 1980). This "cultural-attentional" dysfunction explanation contrasts with an alternative (third) view that alcohol directly and detrimentally influences new learning. According to this idea, a history of protracted alcohol abuse could eventually lead to an extended retrograde amnesia but this would arise as the result of a systematically worsening anterograde problem (Meudell et al., 1980; Brandt and Butters, 1986). Since even mild doses of alcohol are known to impair both recall and recognition memory in normal people (e.g. Lowe, 1981), both of these factors probably have some element of truth in them. Further, chronic alcoholics (not diagnosed as Korsakoffs) show a similar time graded retrograde amnesia but which is less severe than those of Korsakoff amnesics (Albert et al., 1980).

However, a recent case study rules out both the cultural and learning impairment explanations as the sole or even joint contributors to the extent of the retrograde amnesia seen in Korsakoffs; that is, neither together nor alone can these two factors be sufficient to explain all of the retrograde amnesia shown by Korsakoffs.

Butters and Cermak (1986) documented a Korsakoff case (P.Z.), an eminent scientist but chronic alcoholic, who had completed his autobiography some 2 years before becoming amnesic. By sampling items from P.Z.'s autobiography, Butters and Cermak were able to show that he had a dense retrograde amnesia with sparing of remote memories. Since all questions were drawn from his own recently written autobiography this must eliminate the hypothesis that he had never acquired the information: his retrograde problems could not, therefore, be secondary to a deficiency in original learning or through lack of contemporary interest.

Most workers would now probably argue that it is no longer viable to explain the retrograde amnesis in Korsakoffs in terms of a "continuity" with alcohol induced learning deficits.

\section{ALTERNATIVE EXPLANATIONS}

Unless it is assumed that consolidation and storage processes takes decades to complete, then the duration of retrograde amnesia (for example, going back several decades in the case of P.Z.) makes any explanation that the remote memory impairment is attributable to an interruption of storage (as a result of the Wernick-Korsakoff incident) seem an unlikely hypothesis.

Accordingly, a number of alternative accounts have been put forward.

One potential explanation is that, since brain damage is likely to be somewhat less than focal in alcoholic Korsakoffs and might especially involve the frontal lobes (e.g. Jacobsen, 1989), the prolonged retrograde amnesia in these patients could result from cognitive impairments which are incidental to the cause of the retrograde amnesa. One potential cognitive deficit resulting from frontal lobe damage could be a reduction in overall cognitive resources for controlling and modulating all aspects of cognition including memory (Squire, 1986): an alternative characterisation might be a degradation of function reflecting impairments in what Baddeley terms a dys-executive syndrome (e.g. Baddeley and Wilson, 1988).

Using a dual task paradigm we have recently shown that our Korsakoffs do indeed show reduced cognitive resources and a marked inability to monitor, control and share resources in cognitive tasks. In comparison to normal controls and in relation to tasks performed in isolation, they were especially compromised when required to carry out two tasks simultaneously (Meudell et al., 1992). We have yet to ascertain whether this decrease in cognitive capacity relates to the severity of patients' retrograde amnesias but, interestingly, the reduction in resource did not relate to the severity of anterograde amnesis but did show a tendency to correlate with measures of frontal function in our Korsakoffs. The absence of correlation with severity of amnesia is intriguing since it is well known that memory in normal people is severely dis- 
rupted when their cognitive resources are depleted through experimental means (for example, requiring them to engage in distracting tasks during learning). However, it is possible that the diminution of mnemonic function due to pathological reduction in resource in the Korsakoffs is a relatively small factor when compared that due to the (unknown) and individually variable determinant of their amnesia.

Although signs of frontal atrophy per se do not appear to predict (or only weakly predict) remote public or autobiographical memory deficits (Dall'Ora et al., 1989; Kopelman, 1989). Kopelman (1991) has shown that correlations between behavioural measures thought to be sensitive to frontal function do predict remote memory impairments in Korsakoffs (although these same measures did not predict severity of their anterograde impairments).

In any event, these data show that loss of executive control, possibly linked to frontal danage, could indeed be a feature in Korsakoffs.

Baddeley and Wilson (1988) have reported a frontally damaged case in which marked deficits in autobiographical memory were a prominent feature and a number of other studies have examined "event" memory in groups of dementing patients all of whom have putative frontal lobe damage. In Huntingdon's disease (Albert et al., 1981), in Alzheimer's disease (Wilson et al., 1981) and in Parkinson's patients (Freedman et al., 1984) retrograde amnesia extending to decades have been reported.

However, in contrast to Korsakoffs, no evidence for the "sparing" of older memories has been reported in these various dementing groups in whom frontal pathology might be suspected; the forgetting functions for these patients are simply "flattened" in comparison to normal controls. Whilst this is consistent with a non-specific deficit (such as the pathological loss of resource now documented in our patients) causing the retrograde problem, the absence of a temporal gradient in these patients makes it difficult to see how the putative "overlay" of an additional frontal component in Korsakoffs could produce their pattern of remote memory performance: that is, such an overlay could produce a very long retrograde amnesia but not one with a temporal gradient. Accordingly, although a reduction in cognitive resources is a feature in alcoholic induced amnesia, it is not one that can readily explain either the retrograde or the anterograde problems.

Another explanation for the long and temporally graded retrograde amnesia in Korsakoffs relates to the potential explanations for anterograde amnesia. That is, the putative functional causes of anterograde amnesia might have implications for the form of retrograde amnesia.

The first such account stems from Warrington and Weiskrantz's work in the 1970s. They argued that amenesia was caused by a retrieval deficit (attributable to interference resulting from an excess of irrelevant information) and that this would manifest itself not only in amnesics' abilities to retrieve new information but would, necessarily, intrude into the recall of pre-traumatic memories. Sanders and Warrington (1971) claimed that recall from the remote past was simply "flattened" in their amnesics compared to controls. However, this is an isolated result and could have resulted from a floor effect for the patients tested: further, using a very similar news event questionnaire based upon events whose salience was ensured to be equivalent across decades one which has not been repeated in our laboratory (Snowden, 1983): Snowden found a relative preservation of older memories amongst our Korsakoffs.

Although the precise form of Warrington and Weiskrantz's retrieval dificit account is no longer tenable, many of the other accounts the retrograde amnesia in Korsakoff's disease implicitly point to some form of retrieval deficit. In this regard, Shallice (1986) has argued that consistency of responding over time is an important marker in distinguishing between storage and retrieval failures. In particular, he argues that "item specific" consistency of responding over time could represent a storage failure whist variability between one occasion and another in response to the same question could reflect a retrieval failure (he argues that an "item" recalled on one occasion but not another could not reflect a storage failure). In fact, since Shallice assumes an unproved theory of memory that is probably wrong, some have disputed that storage or retrieval failures can be distinguished in terms of consistency of responses (e.g. Mayes, 1988). However, it is interesting to note that whilst the post-encephalitic patient S.S. made inconsistent recall from time to time (Cermak and O'Connor, 1983), Korsakoffs have been reported to show consistent responses over separate testing sessions (Pickering, 1988). However, another report has shown that a further post-encephalitic does show consistency of responding over time (Warrington and McCarthy, 1988); this variability within patients of the same encephalitic etiology could suggest that the precise pattern of responding over time is a feature that is irrelevant or incidental to the root of the retrograde or anterograde impairment. What consistency of responding might relate to is unknown but it is tempting to speculate that it could link to frontal damage given the propensity of some frontal patients to show preservation of response in many situations whilst other frontals, manifesting unpredictable responses, tend to show more or less flamboyant confabulation when confronted with autobiographical tests (see Baddeley and Wilson, 1988). Notwithstanding Pickering's observations and given the wide variety in extent and severity of frontal involvement in Korsakoffs, similar heterogeneity in consistency of responding over time amongst these amnesics might also be expected. Correlations amongst measures of mnemonic consistency and frontal indicants of preser- 
vation or confabulation-like tendencies could prove informative in this respect.

One of the earliest views about the cause of memory problems in amnesia is Butters and Cermak's semantic coding deficit account-they argued that Korsakoffs spontaneously failed to employ semantic strategies during learning and retrieval. Since "striving for meaning" is an important determiner of good memory, Butters and Cermak suggested that the absence of semantic analysis in amnesics caused their memory problems. Such a deficit would explain an extended retrograde amnesia (as a consequence of a semantically based retrieval strategy failure) but would not explain why older memories are spared in Korsakoffs. In fact, this view is almost certainly incorrect on other grounds since many Korsakoff patients can show perfectly normal semantic processing strategies in the face of dense amnesia (see Meudell and Mayes, 1982 for review).

Another explanation for the preservation of older memories in Korsakoffs is that there are two subsystems involved in retrieving memories. Specifically, it has been suggested that semantic memory (a system involved in storing meaning and knowledge about facts) might be preserved in amnesics but that episodic memory (a system involved in memory for day-to-day events) is compromised (e.g. Tulving, 1983). Evidence in support of preserved semantic memory stems from observations that vocabulary can be normal in amnesics and that reaction times to semantic tasks are also in the normal range (e.g. Meudell et al., 1980).

The distinction between episodic and semantic memories does not help the explanation of the temporal gradient in Korsakoff's disease unless an additional assumption is made about the nature of the preserved (older) memories.

The preservation of older personal memories is attributed to these being, in effect, represented in the putative semantic store rather than the episodic one; all stored information, it is argued, tends to become "semanticised" over time (see Cermak, 1984). However, amnesics can recall, when pressed, very remote memories of a personal, autogiographical or "episodic" type (Zola-Morgon et al., 1983). Of course it could be argued that even apparently "episodic" information could become, over time, represented in a semantic system in much the same way as our knowledge of the meaning of words: although possible, there is no independent evidence at the moment that this might occur and the explanation is presently in danger of circularity.

One of the most recent and influential views about the functional cause of amnesia is the context deficit account (see Mayes et al., 1985). This view suggests that the anterograde deficit in Korsakoffs might stem from a failure to employ contextual features when remembering. Since, it is argued, contextual information is necessary for good recall and recognition, its pathological absence will lead to poor memory. As it stands, such an account would predict a long retrograde amnesia but, in the absence of additional assumptions would not predict the sparing of older memories. However, Parkin et al. (1991) have suggested that, as memories age they become "decontextualised". That is, whilst newly acquired information needs to be retrieved by its contextual markers (where and when something happened for example), as memories become more and more retrieved and reviewed, access to them becomes less dependent upon these contextual features. This "decontextualisation" of old memories is, of course, formally similar to Cermak's hypothesis about the possible "sementicisation" of aged memories. In any event, decontextualisation might lead to the sparing of older memories since they might be processed and retrieved by systems not damaged in amnesia.

This two factor account of the duration and form of Korsakoffs retrograde amnesia is attractive but it too appears to be false. Parkin et al. (1990) used contextual cues to investigate the hypothesis. They reasoned that if older memories are decontextualised in some way then providing contextual "hints" might not help older memories but might well aid the recall or recognition of newer memories which, by hypothesis, should be dependent upon contextual features for their efficient retrieval. By using photographs of famous people without context (for example, Elvis Presley without a guitar and all his other accoutrements) and with context (Presley ready for the stage) they found that, when subjects were asked to identify the people in the photographs, normal individuals benefited equally from contextual prompts however old the memory being assessed. Contrary to the context deficit account they also found that, for Korsakoff amnesics, contextual hints gave a disproportionate improvement in recall the older the memory. The relative amnesic sensitivity of older memories to contextual information and the relative insensitivity of newer memories to context strongly argues against the hypothesis of progressive "decontextualisation" of memories as they age.

This study clearly needs repeating, however, since it is puzzling why new amnesic memories do not benefit from contextual cueing. In addition, the kind of "interactive" and semantically based context employed by Parkin could be inappropriate if the relevant contextual retrieval processes putatively impaired in amnesia involve more specific spatio-temporal information.

In any event, these and other data have led Parkin (1991) to put forward a radically different explanation for anterograde and retrograde amnesia in Korsakoffs. He suggests that the functional explanations for anterograde and retrograde are different (and so reflect damage to independent neurological structures). Parkin's view is that the anterograde amnesia represents a contextual coding defi- 
cit whilst the retrograde problem reflects storage problems: the absence of correlation between anterograde and retrograde severity is of course weak support in this regard (e.g. Shimamura and Squire, 1986).

However, there may be other explanations for Parkin's data that do not necessarily imply a separation of the processes involved in new learning from those mediating retrieval of old, remote memories.

Whilst contextual processing impairments could relate to the cause of anterograde amnesia, there is some evidence that the contextual processing deficits might be attributable to incidental frontal damage in Korsakoffs (e.g. Mayes et al., 1985). It could be argued, therefore, that such processing plays no role in the causation of retrograde amnesia since it is actually quite unconnected with the mechanisms of the anterograde deficit. Put differently, Parkin assumes that the anterograde deficit is attributable to a context processing problem; however, if anterograde amnesia is not causally linked to deficiencies in processing context, then apparently anomalous contextual effects in retrograde amnesia may have no necessary theoretical importance.

If this "frontal" account were true it would not, of course, explain exactly what is compromised in amnesia; however, it would mean that it might not be necessary to adopt (a possibly unparsimonious) two factor account for the explanation of anterograde and retrograde amnesias.

Nevertheless, there is now an accumulating body of evidence-based upon single case studies of a non-alcoholic etiology-that severe retrograde amnesia can present in the absence of significant anterograde deficits. As a result, the idea that the anterograde and retrograde deficits in the Wernicke-Korsakoff syndrome might have different explanations (whatever these might be) might deserve to be taken seriously.

\section{DISSOCIATIONS BETWEEN RETROGRADE AND ANTEROGRADE AMNESIA}

Goldberg et al. (1981) studied a patient who developed amnesia following head injury. Although he initially presented with both retrograde and anterograde amnesia, over the course of time his anterograde deficits resolved leaving a profound 20 years retrograde amnesia for public events as well as autobiographical details. Similarly, Andrews et al. (1982) reported a case with a retrograde deficit spanning some 40 years in the face of minimal deficits in new learning abilities. Stuss and Guzman (1988) have also described a patient whose mild difficulties in new learning contrasted sharply with his poor recall of public events and a practically total failure to remember any autogiographical information.

One problem with these studies is the suggestion that, even in the presence of demonstrable brain damage, there could be an overlay of a psychogenic or "functional" component (see Mayes, 1988). This issue is a serious one since, for example, it has recently been reported that Stuss's case recovered totally during an amytal test and must, therefore, have had memory problems of a psychogenic sort despite demonstrable pathology (Stuss and Guzman, 1988).

However, there are a number of other cases where this criticism may not apply. Kapur et al. (1986) have reported a case of transient global amnesia where a marked retrograde amnesia could be demonstrated in the face of normal (and in certain cases) supernormal anterograde performance; Kapur et al. (1989) have also been able to show this pattern has persisted over a 5-year testing period. The pattern of the patient's anterograde performances almost certainly rules a functional contribution so the data from this patient strongly implies a potential dissociation between anterograde and retrograde problems.

More recently, O'Connor et al. (1992) have reported a single case of encephalitis-induced amnesia who also demonstrated a severe retrograde amnesia for autobiographical details and for public events; however, her anterograde impairment was very mild in comparison. There is no suggestion of a functional overlay in this well documented case of encephalitis and so her data too point towards the separation of anterograde and retrograde deficits.

In a similar case de Renzi (1992) has recently described an anoxic amnesic who had dense retrograde amnesia for autobiographical and "fact" memory covering most of the pretraumatic period; in contrast the patient's ability to retain learn new information appeared perfectly intact. This case did, however, show faster forgetting of this newly acquired information over very long time periods (weeks and months) compared to controls.

If the neurological bases of retrograde and anterograde deficits are indeed separate (and so might be independently compromised) it should be possible to observe cases of the opposite dissociation where patients have marked problems with learning new information but in whom little or no retrograde amnesia is detectable. Regard and Landis (1984) have reported two such cases involving atypical transient global amnesia. The course of recovery from TGA in these patients showed a progressive ability to recall remote memories (retrograde amnesia had resolved) whilst anterograde deficits persisted. One problem with the interpretation of these data is that they could simply reflect the different rates of recovery of retrograde and anterograde amnesia. That is, the manifestation in mnemonic performance of recovery of a common system underlying retrograde and anterograde deficits might be different for old and new learning. However, Zola-Morgan et al. (1986) have shown that the amnesic patient R.B.-who had memory problems resulting from clipping 
of the anterior communicating artery and in whom differential recovery rates cannot be an issue-had a virtually undetectable retrograde amnesia: however, his anterograde problems appear to be mild-moderate. Since some other patients-following surgery of the anterior communicating artery-show retrograde amnesias spanning decades (Gade and Mortensen, 1990), it is unlikely that R.B.'s preserved remote memory reflects the general functional consequences of the lesion involved in this group of patients. Of course since R.B. was reported as showing a mild anterograde amnesia an explanation for his preserved remote memory in terms of a generally mild problem with memory remains possible.

The discussion in this section has necessarily centred around amnesic patients of a non alcoholic etiology. However, as noted above, little correlation between retrograde and anterograde severity has been reported within alcoholic patients, thus supporting the notion that they could result from independent impairments. In this connection, it is important note that, whilst in rank order terms, Korsakoffs may show rankings of anterograde and retrograde problems which are uncorrelated, the reported retrograde amnesia in these patients appears always to be of a long duration. To my knowledge (and unlike patients with amnesia resulting from surgery to the anterior communicating artery) no Korsakoff has been reported who shows severe anterograde deficits in the face of a steeply graded-and brief-retrograde amnesia. Yet this is exactly what, in principal, should occur if Parkin (1991) is right that retrograde and anterograde amnesia in the Wernicke-Korsakoff syndrome have different explanations.

The view that anterograde and retrograde amnesias are dissociable impairments is intriguing but remains, as yet, unproven. However, reports of a series of "clean" Korsakoffs (without frontal or other cortical damage) who separately present with (a) steeply graded and brief retrograde amnesia along with an anterograde amnesia, (b) no retrograde amnesia in the face of severe anterograde problems, and (c) steeply graded and brief retrograde amnesia in the absence of anterograde deficits, would significantly buttress the hypothesis. Korsakoffs without frontal signs are rare but do exist; just how plausible are the other expectations remains an open issue.

\section{CONCLUSION}

Of course, even if it were eventually proved that anterograde and retrograde amnesia could arise as dissociable impairments (and which, in Korsakoffs, might frequently tend to arise "hand in hand" through multiple lesions), this fails to provide any clue as to why retrograde amnesia takes the form that it does and so the explanation for the profound length of the retrograde amnesia and its temporal gradient would remain obstinately obscure.
That is, any theory of retrograde amnesia alone or one which encompassed both retrograde and anterograde grade amnesia, must explain the prolonged temporal gradient invariably noted in Korsakoffs.

\section{REFERENCES}

Albert MS, Butters N and Levin J (1979) Temporal gradients in the remote memory of patients with alcoholic Korsakoff's disease. Archives of Neurology, 36, 211-216.

Albert MS, Butters N and Brandt J (1980) Memory for remote events in alcoholics. Journal for the Study of Alcohol, 41, 1071-1081.

Albert MS, Butters N and Brandt J (1981) Patterns of remote memory in amnesic and dementing subjects. Archives of Neurology, 38, 495-500.

Andrews E, Poser CM and Kessler M (1982) Retrograde amnesia for forty years. Cortex, 18, 441-458.

Baddeley AD and Wilson B (1986) Amnesia, autobiographical memory and confabulation. In: Autobiographical Memory (Ed. D Rubin). Cambridge University Press, Cambridge.

Baddeley AD and Wilson B (1988) Frontal amnesia and the dysexecutive syndrome. Brain and Cognition, 7, 212-230.

Brandt J and Butters N (1986) The alcoholic Wernicke-Korsakoff syndrome and its relationship to long term alcohol abuse. In: Neuropsychological Assessment of Neuropsychiatric Disorders (Eds I Grant and KM Adams). Oxford University Press, New York.

Butters N and Cermak LS (1986) A case study of the forgetting of autobiographical knowledge: implications for the study of retrograde amnesia. In: Autobiographical Memory (Ed. D Rubin). Cambridge University Press, Cambridge.

Cermak LS (1984) The episodic/semantic distinction in amnesia. In: The Neuropsychology of Memory (Eds LR Squire and N Butters). Guildford Press, New York.

Cermak LS and O'Connor M (1983) The anterograde and retrograde retrieval ability of a patient with amnesia due to encephalitis. Neuropsychologia, 21, 213-234.

Crovitz HF and Schiffman H (1974) Frequency of episodic memories as a function of their age. Bulletin of the Psychonomic Society, 4, 517-518.

Dall'Ora P, Sala SD and Spinnler H (1989) Autobiographical memory: its impairment in amnesic syndromes. Cortex, 25, 197-217.

Eich JE (1980) The cue-dependent nature of state-dependent retrieval. Memory and Cognition, 8, 157-173.

Freedman M, Rivoira P, Butters N, Sax DS and Feldman RG (1984) Retrograde amnesia in Parkinson's disease. Canadian Journal of Neuroscience, 11, 297-301.

Gade A and Mortensen L (1990) Temporal gradient in the remote memory impairment of amnesic patients with lesions of the basal forebrain. Neuropsychologia, 28, 985-1001.

Goldberg E, Antin SP, Bilder RM, Hughes JEO and Mattis S (1981) Retrograde amnesia: possible role of mesencephalic reticular activation in long-term memory. Science, 213, 1392-1394.

Jacobsen RR (1989) Alcoholism in Korsakoff's syndrome and the frontal lobes. Behavioural Neurology, 2, 25-38.

Kapur N, Heath P, Meudell P and Kennedy P (1986) Amnesia can facilitate memory performance: evidence from a patient with dissociated retrograde amnesia. Neuropsychologia, 24, 215-221.

Kapur N, Young A, Bateman D and Kennedy P (1989) Focal 
retrograde amnesia: a long term and clinical neuropsychological follow-up. Cortex, 25, 387-402.

Kinsbourne M and Wood F (1982) Theoretical considerations regarding the episodic-semantic memory distinction. In: Human Memory and Amnesia (Ed. LS Cermak). Lawrence Erlbaum, Hillsdale, NJ.

Kopelman M (1989) Remote and autobiographical memory, temporal context memory and frontal atrophy in Korsakoff and Alzheimer patients. Neuropsychologia, 27, 437-460.

Kopelman MD (1991) Frontal dysfunction and memory deficits in the alcoholic Korsakoff syndrome and Alzheimer-type dementia. Brain, 114, 117-137.

Lowe G (1981) State dependent recall with moderate doses of alcohol. Current Psychological Research, 1, 3-8.

Mackinnon DF and Squire LR (1989) Autobiographical memory and amnesia. Psychobiology, 17, 247-256.

Marslen-Wilson WD and Teuber HL (1975) Memory for remote events in anterograde amnesia: recognition of public figures from news photographs. Neuropsychologia, 13, 353-364.

Mayes AR (1988) Human Organic Memory Disorders. Cambridge University Press, Cambridge.

Mayes AR, Meudell PR and Pickering A (1985) Is organic amnesia caused by a selective deficit in remembering contextual information? Cortex, 21, 167-202.

Mayes AR, Meudell PR, Mann and Pickering A (1988) Location of lesions in Korsakoff's syndrome:neuropsychological and neuropathological data on two patients. Cortex, 24, 367-388.

Meudell PR and Mayes AR (1982) Normal and abnormal forgetting: some comments on the human amnesic syndrome. In: Normality and Pathology in Cognitive Function (Ed. AW Ellis). Academic Press, London.

Meudell PR, Northen B, Snowden JS and Neary D (1980) Long term memory for famous voices in amnesic and normal subjects. Neuropsychologia, 18, 133-139.

Meudell PR, Mayes AR and Neary D (1980) Amnesia is not caused by cognitive slowness. Cortex, 16, 413-419.

Meudell PR, Mayes AR and MacDonald C (1992) Dual task performance in amnesic and normal people. In preparation

O'Connor M, Butters N, Miliotis P and Eslinger P (1992) The dissociation of anterograde and retrograde amnesia in a patient with herpes encephalitis. Journal of Clinical and Experimental Neuropsychology, 14, 159-178.

Ogden J and Corkin S (1991) Memories of H.M. In: Memory Mechanisms: A Tribute to G.V. Goddard (Eds WC Abraham, MC Corballis and KG White). Lawrence Erlbaum, New York.

Parkin AJ, Montaldi D and Leng NR (1990) Contextual cueing effects in the remote memory of alcoholic Korsakoff patients and normal subjects. Quarterly Journal of Experimental Psychology, 42, 585-596.

Parkin AJ (1991) The relationship between anterograde and retrograde amnesia in alcoholic Wernicke-Korsakoff syndrome. Psychological Medicine, 21, 11-14.
Pickering A (1988) Cited by Mayes (1988), op cit.

Regard M and Landis T (1984) Transient global amnesia: neuropsychological dysfunction during attack and recovery in two "pure" cases. Journal of Neurology, Neurosurgery and Psychiatry, 47, 668-672.

Renzi E de (1992) Focal retrograde amnesia: a PET study. Paper presented at Anatomy of Amnesia Meeting, Manchester.

Ribot TA (1882) The Diseases of Memory. Appleby, New York.

Sagar HJ, Cohen NJ, Corkin S and Growdon JH (1985) Dissociations among processes in remote memory. Annals of the New York Academy of Sciences, 444, 533-535.

Sanders HI and Warrington EK (1971) Memory for remote events in amnesic patients. Brain, 94, 661-668.

Shallice T (1986) Impairments of semantic processing: multiple dissociations. In: The Cognitive Psychology of Language (Eds $M$ Coltheart, M Job and G Sartori). Lawrence Erlbaum, Hillsdale, NJ.

Shimamura A and Squire LR (1986) Korsakoff's syndrome: a study of the relation between anterograde amnesia and remote memory impairment. Behavioral Neuroscience, 100, $165-170$

Snowden JS (1983) Cognitive disorders in alcoholic amnesia. Ph.D thesis, University of Manchester.

Squire LR (1986). Mechanisms of memory. Science, 232 1612-1619.

Squire LR and Cohen NJ (1984) Human memory and amnesia. In: PsychoBiology of Learning and Memory (Eds G Lynch, JL McGaugh and NM Weinberger). Guildford Press, New York.

Squire LR and Slater PC (1978) Anterograde and retrograde memory impairment in chronic amnesia. Neuropsychologia 16, 313-322.

Squire LR, Haist F and Shimamura AP (1989) The neurology of memory: quantitative assessment of retrograde amnesia in two groups of amnesic patients. Journal of Neuroscience, 9 , 828-839.

Stuss DT and Guzman A (1988) Severe remote memory loss with minimal retrograde amnesia: a clinical note. Brain and Cognition, 8, 21-30.

Tulving E (1983) Elements of Episodic Memory. Clarendon Press, Oxford.

Warrington EK and McCarthy RA (1988) The fractionation of retrograde amnesia. Brain and Cognition, 7, 184-200.

Weiskrantz L (1985) On issues and theories of the human amnesic syndrome. In: Memory Systems of the Brain (Eds JL McGaugh and G Lynch). Guildford Press, New York.

Zola-Morgan S, Cohen NJ and Squire LR (1983) Recall of remote episodic memory in amnesia. Neuropsychologia, $\mathbf{2 1}$ 487-500.

Zola-Morgan S, Squire LR and Amaral DG (1986) Human amnesia and the medial temporal region: enduring memory impairment following a bilateral lesion limited to filed CA1 of the hippocampus. Journal of Neuroscience, 6, 2950-2967. 


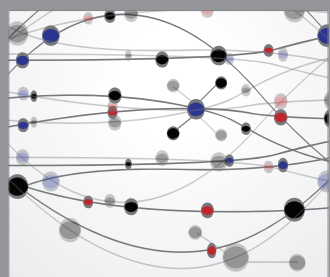

The Scientific World Journal
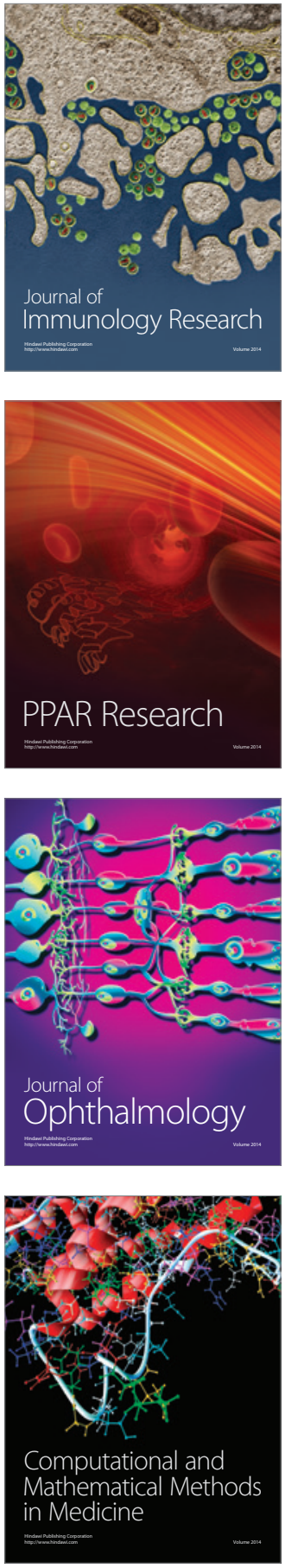

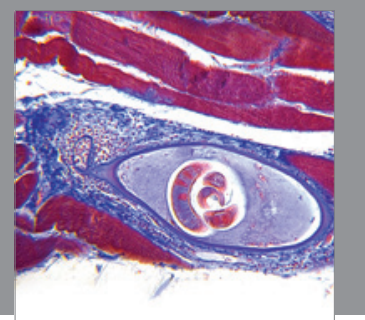

Gastroenterology

Research and Practice
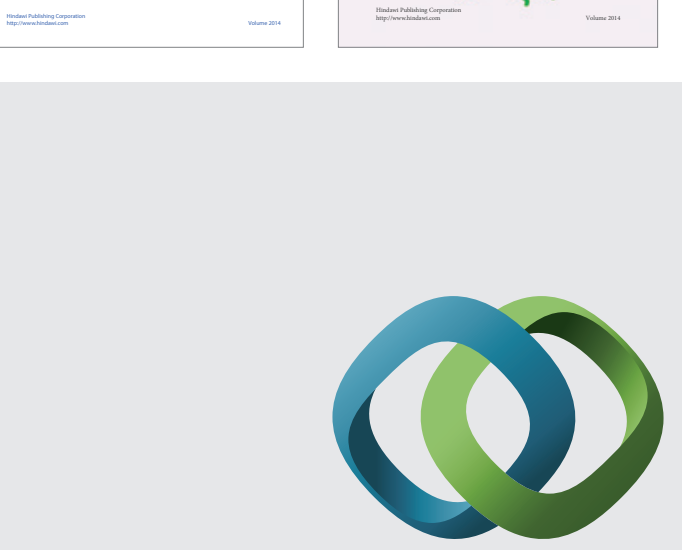

\section{Hindawi}

Submit your manuscripts at

http://www.hindawi.com
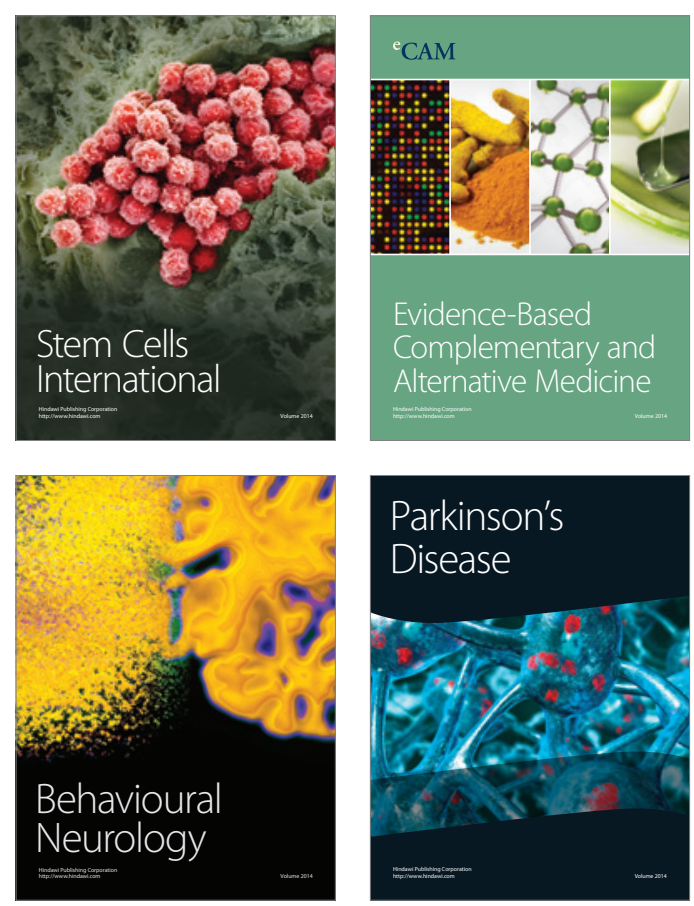

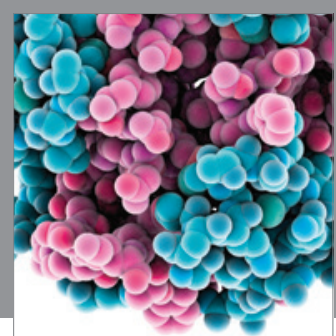

Journal of
Diabetes Research

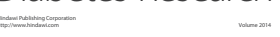

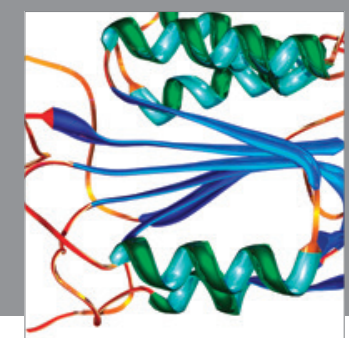

Disease Markers
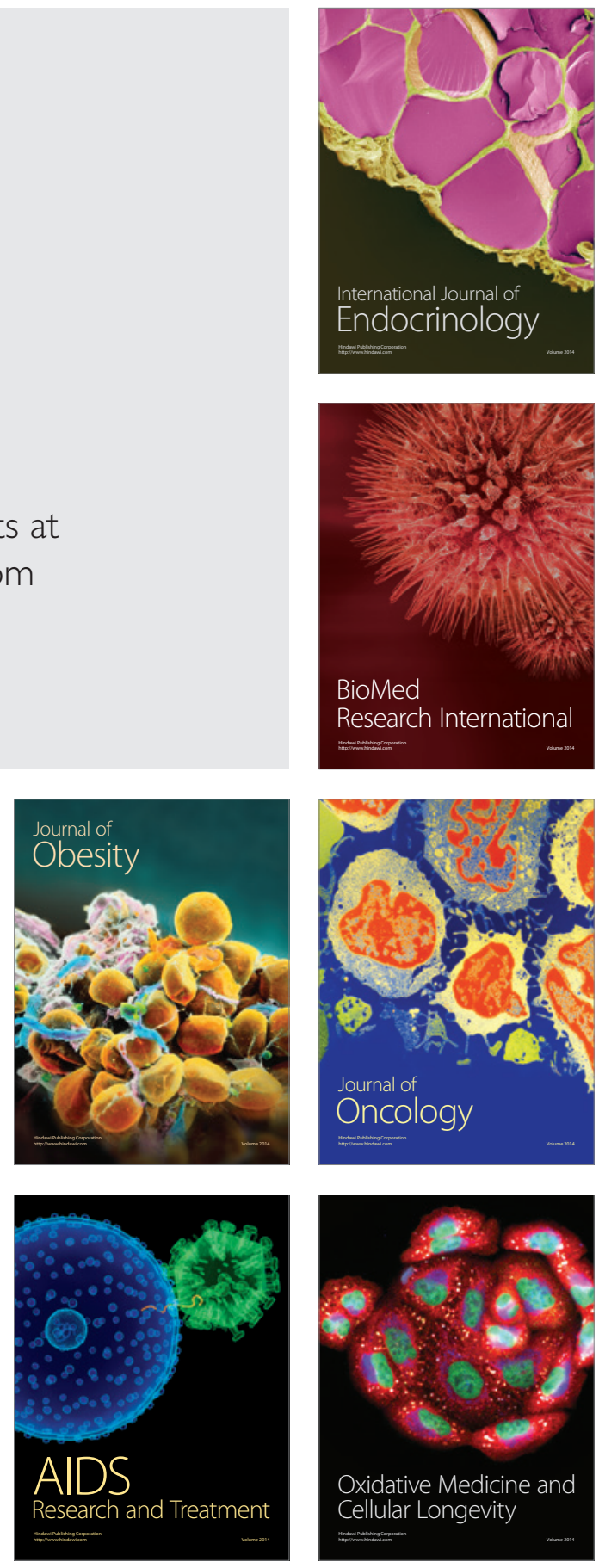